\section{A) Check for updates}

Cite this: Food Funct., 2022, 13, 2570

\title{
Oral administration of Lactobacillus delbrueckii enhances intestinal immunity through inducing dendritic cell activation in suckling piglets $\uparrow$
}

\author{
Wei Peng, ${ }^{a, b}$ Ying-Hui Li, ${ }^{a, b}$ Guan Yang, ${ }^{c}$ Jie-Lin Duan, (D) a,b Ling-Yuan Yang, ${ }^{a, b}$ \\ Li-Xiang Chen, ${ }^{a, b}$ Shu-Ling Hou ${ }^{a, b}$ and Xing-Guo Huang ${ }^{\star a, b}$
}

\begin{abstract}
Lactobacillus delbrueckii (LAB) has been demonstrated to exert versatile beneficial effects on modulating intestinal immunity, increasing gut microbial diversity, promoting growth performance, and even preventing disease onset in pigs. However, the underlying mechanism of LAB-mediated gut immunity regulation in piglets remains unclear. In this study, we found that supplementation of LAB significantly increases serum TNF- $\alpha$, ileum IL-4, and IL-10 levels compared with the control group. Meanwhile, oral supplementation of $L A B$-modified gut microbial communities was evidenced by the increased abundance of the Lactobacillus genus in the colon. Mechanistically, LAB induced dendritic cell (DC) maturation and activation, which may be relevant to the activation of NF-кB and MAPK signaling pathways. Moreover, we found that oral administration of LAB during the suckling period shows long-lasting immunomodulatory impacts on intestinal immunity after weaning. Collectively, this study uncovers the mechanism of LAB in regulating the intestinal immunity of piglets, suggesting that $L A B$ can be developed as an immunoenhancing biological agent during the suckling period.
\end{abstract}

Received 2nd December 2021 Accepted 12th January 2022 DOI: $10.1039 / \mathrm{d} 1 \mathrm{fo} 03864 \mathrm{~h}$ rsc.li/food-function immune system and enhance piglet immunity is important and necessary for pig production.

Probiotics have been attracting increasing attention due to their immunomodulatory function with few side effects. Many studies have demonstrated the beneficial effects of Lactobacillus on the maintenance of gut homeostasis. ${ }^{4,5}$ As a normal resident microflora in the gastrointestinal tract of humans and animals, the colonization and abundance of Lactobacillus contribute to the development and function of intestinal immune cells, and thus could further impact the pathogenesis of intestinal diseases such as inflammatory bowel disease (IBD).$^{6-8}$ For example, Lactobacillus has been reported to regulate the differentiation of $\mathrm{CD} 4^{+} \mathrm{CD} 8 \alpha \alpha^{+} \mathrm{T}$ cells ${ }^{9}$ and $\mathrm{CD} 4{ }^{+} \mathrm{FoxP}^{+}{ }^{+} \mathrm{T}$ regulatory cells, ${ }^{10}$ increase the numbers and effector functions of distinct B cell subsets in Peyer's patches (PPs) ${ }^{11}$ and promote the maturation and function of dendritic cells (DCs) and macrophages. ${ }^{10,12}$ Mechanistically, probiotics alleviate intestinal inflammation via modulation of toll-like receptor (TLR)-myeloid differentiation factor 88 (MyD88)-dependent mitogen-activated protein kinase (MAPK) and nuclear factor kappa-B (NF-kB) signaling. ${ }^{13-15}$ Therefore, using probiotics including Lactobacillus to enhance intestinal immunity and health has a promising prospect.

Lactobacillus delbrueckii (LAB), which is a Lactobacillus species, plays various beneficial probiotic roles in humans and animals. Our previous study has shown that LAB enhances 
anti-oxidant capacities, increases gut microbial community, and decreases diarrhea rates, and consequently improves the growth performance of pigs. ${ }^{16-20}$ In addition, we found that LAB could enhance intestinal mucosal cytokine and secretory IgA (sIgA) production in piglets. ${ }^{16,19}$ However, the underlying mechanism of LAB-mediated intestinal immunity regulation remains unclear.

Here, we show that oral administration of $\mathrm{LAB}$ increases immune-related cytokine production and induces DC maturation and activation in suckling piglets, which is related to the activation of MAPK and NF- $\kappa$ B signaling. Notably, we found that the immunomodulatory properties of LAB during the suckling period exhibit long-term effects on intestinal immunity during the weaned period. Our data revealed the potential mechanism of LAB in regulating intestinal immunity, suggesting that LAB can be developed as an immunoenhancing biological feed additive.

\section{Materials and methods}

\subsection{Preparation and culture of LAB}

The strain Lactobacillus delbrueckii (LAB, CCTCC M 207040) used in this study was preserved in the microbiology laboratory of the College of Animal Science and Technology, Hunan Agricultural University. LAB was cultured at $37{ }^{\circ} \mathrm{C}$ for $48 \mathrm{~h}$ in DeMan, Rogosa, and Sharp (MRS) medium under anaerobic conditions. The viable counts in the culture medium were determined by the gradient dilution coating method based on our previous study, ${ }^{16}$ and then adjusted to $5 \times 10^{9}$ colonyforming units (CFU) per mL. An appropriate dose of LAB was applied for oral gavage to suckling piglets. For cell stimulation, LAB was heat-killed at $56{ }^{\circ} \mathrm{C}$ for $60 \mathrm{~min}$.

\subsection{Animals and experimental design}

To determine the effect of $\mathrm{LAB}$ supplementation on intestinal immunity during the suckling period, eighty neonatal piglets (Duroc $\times$ landrace $\times$ large Yorkshire) from 10 litters were randomly assigned to the control group (CON) and LAB group (LAB) based on the litter origin and body weight (BW), and each group has 5 litters with 8 piglets ( 4 females and 4 males). The piglets from the LAB group were intragastrically administered with $\mathrm{LAB}$ at amounts of $1,2,3$, and $4 \mathrm{~mL}\left(5 \times 10^{9} \mathrm{CFU}\right.$ per $\mathrm{mL}$ per pig) at $1,3,7$, and 14 days of age, respectively. The piglets from the CON group received the sterilizing saline. At 21 days of age, 6 piglets in each group were sacrificed for sample collection.

To test whether $\mathrm{LAB}$ treatment during the suckling period exhibited long-lasting effects on intestinal immunity after weaning, we treated suckling piglets with LAB as above. After the piglets were weaned at 21 days of age, the piglets from the CON and LAB groups were randomly divided into 6 pens (3 pens per group) with 5 pigs in each pen. These weaned piglets were fed with creep feed and managed until 49 days of age. Among the experimental period, 6 piglets were sacrificed at 28 and 49 days of age for sample collection.
All suckling piglets had free access to sow milk and drinking water, and were housed in an environmentally controlled farrowing cage with hard plastic slatted flooring. All weaned piglets were housed in a temperature-controlled nursery and had ad libitum access to feed and water. The creep diet was corn-soybean meal-based as reported in our previous study. $^{16}$

All samples were collected and prepared based on our previous study. ${ }^{18}$ Briefly, the serum samples were collected by venipuncture into $10 \mathrm{ml}$ tubes and centrifuged at $3000 \mathrm{~g}$ for $10 \mathrm{~min}$ at $4^{\circ} \mathrm{C}$. About $2 \mathrm{~g}$ of colonic contents were taken from the middle of the colon from the piglets for analysis of microbial diversity. Segments $(8 \mathrm{~cm})$ of the jejunum and ileum were flushed with ice-cold phosphate-buffered saline thoroughly for mucosal sample preparation.

\subsection{DC2.4 cells culture and stimulation}

DC2.4 cells (the dendritic cell line) were cultured in RPMI-1640 medium supplemented with 10\% FBS (fetal bovine serum) and antibiotics (100 $\mathrm{U} \mathrm{mL}^{-1}$ penicillin and $100 \mu \mathrm{g}$ $\mathrm{mL}^{-1}$ streptomycin) at $37^{\circ} \mathrm{C}$ in a $5 \% \mathrm{CO}_{2}$ incubator.

In the cell stimulation experiment, $1 \times 10^{6}$ DC2.4 cells were plated in 6-well cell culture plates. The cells were stimulated with lipopolysaccharides (LPS), a low dose of heat-killed (LAB-HK-L), live (LAB-L) LAB $\left(1 \times 10^{6}, \mathrm{MOI}=1\right)$, a high dose of heat-killed (LAB-HK-H), or live $(\mathrm{LAB}-\mathrm{H}) \mathrm{LAB}\left(1 \times 10^{7}\right.$, MOI $=$ 10). Then, the cells were collected at indicated time points for further analysis.

\subsection{Enzyme-linked immunosorbent assay (ELISA) for cytokine measurements}

After LPS, heal-killed, or live LAB stimulation for 24 hours, the supernatants of the DC2.4 cells were collected for the ELISA assay. The jejunal and ileal mucosa were homogenized with ice-cold normal saline. After centrifugation $(3000 \mathrm{~g}$ for $10 \mathrm{~min}$ at $4{ }^{\circ} \mathrm{C}$ ), the supernatant of each sample was collected for the ELISA assay. The protein levels of TNF- $\alpha$, IL-12, IL-2, IL-10, IFN- $\gamma$, CCL20, CX3XL1, and IL-4 were measured by using ELISA kits (ELISA kit's information is listed in ESI Table $1 \dagger$ ) according to the manufacturer's protocol.

\subsection{Flow cytometry}

Fluorescence-coupled antibodies for flow cytometry including FITC-MHC-II, PE-CD80, and APC-CD86 were obtained from Abcam Plc. After LPS, heat-killed, or live LAB stimulation, DC2.4 cells were collected and washed. For surface staining, the cells were washed and stained with fluorescence-conjugated antibodies for 30 minutes at $4{ }^{\circ} \mathrm{C}$. After being washed with PBS, the cells were run on a BD LSRFortessa (BD Immunocytometry Systems), and data were analyzed with FlowJo (Tree Star).

\subsection{Quantitative real-time PCR}

Total RNA from DC2.4 cells, jejunum, or ileum tissues was isolated using TRIzol (Invitrogen) and then treated with DNase I 
(Invitrogen, USA). A total of $1 \mu \mathrm{g}$ of RNA was transcribed using PrimeScript $^{\mathrm{TM}}$ RT Master Mix (Takara, RR036A). RT-PCR was performed using Power SYBR Green PCR Master Mix (TaKaRa, RR420A). The amounts of the transcript were normalized to those of the house-keeping gene Gapdh. The primers used in this study are listed in ESI Table $2 . \dagger$

\subsection{Western blot}

Approximately $100 \mathrm{mg}$ of each jejunum and ileum sample were homogenized and then lysed in ice-cold radio-immunoprecipitation assay (RIPA) lysis buffer (Beyotime Institute of Biotechnology, Beijing, China) supplemented with phosphatase inhibitor and protease inhibitor cocktail tablets (Roche Diagnostics Ltd, Shanghai, China) based on a previous report. $^{21}$

DC2.4 cells were stimulated with LPS, heal-killed, or live LAB, and the cells were collected with RIPA lysis buffer. After centrifugation $\left(12000 \mathrm{~g}\right.$ for $10 \mathrm{~min}$ at $4{ }^{\circ} \mathrm{C}$ ), the supernatants were collected for further analysis.

Approximately $50 \mu \mathrm{g}$ protein of each sample was used for western blot analysis based on a previous study. ${ }^{22}$ The primary and secondary antibodies used in this study are listed in ESI Table $3 . \dagger$

\subsection{S rRNA sequencing for analyzing the microbiota composition}

The colonic contents were collected after the piglets were sacrificed. Total genome DNA from each colonic sample was extracted according to the previous study. ${ }^{23}$ After detection by $1 \%$ agarose gel electrophoresis, the extracted genome DNA was sent to Shanghai Personalbio Technology Co., Ltd for $16 \mathrm{~S}$ rRNA sequencing. After amplification using a specific primer with the barcode (16S V3 + V4), sequencing was performed on the Illumina Miseq platform. A phylogenic tree and OTU table were obtained from the Mothur Bayesian classifier. Sequencing libraries were generated and sequencing data were processed according to the previous study. ${ }^{5,24}$ Principal Coordinate Analysis (PCoA) was performed to get principal coordinates and visualize from the complex, multidimensional data. The indices of Shannon, Simpson, Chao1, and ACE were used to evaluate the complexity of species' alpha diversity. Predicted functional analysis of the colonic microbiota was performed by PICRUSt (Phylogenetic Investigation of Communities by Reconstruction of Unobserved States). The OTUs, alpha diversity, and beta diversity analysis, and microbiota structural analysis were done according to our previous study. ${ }^{25}$ Statistical comparison of the relative abundance of the colonic microbiota was performed using a two-tailed unpaired Student's $t$-test to identify the differences in bacterial taxa between the CON and LAB groups. The analysis procedures of the colonic microbiota were processed via the Majorbio Cloud Platform (Shanghai Personalbio Technology Co., Ltd, China). The raw reads in this study were uploaded to the National Center of Biotechnology Information (NCBI) Sequence Read Archive (SRA) database under accession number PRJNA792735.

\subsection{Statistical analysis}

All values in the paper are given as means \pm SEM unless stated otherwise. Significant differences between the two groups were analyzed with a two-tailed unpaired $t$-test. Multiple groups were analyzed by one-way analysis of variance (ANOVA). All statistical analyses were performed using the GraphPad Prism software (GraphPad). Statistical significance was set on the basis of the $P$-value. N.S., $P>0.05 ;{ }^{*} P<0.05,{ }^{*} P<0.01$, ${ }^{* * *} P<0.001$.

\section{Results}

\subsection{Oral administration of LAB enhances intestinal immunity in suckling piglets}

Many studies have shown that probiotics improve intestinal immunity. ${ }^{6}$ Consistent with previous reports, we found that the oral supplementation of LAB (Fig. 1A, experimental design) significantly increases the serum TNF- $\alpha$ and IgA levels, while having no impacts on the serum IL-2 and IFN- $\gamma$ levels compared with the CON group (Fig. 1B). Next, we assessed immune responses in jejunum and ileum from the piglets treated with LAB. We observed that the LAB treatment exhibits no effects on TNF- $\alpha$, IFN- $\gamma$, IL-2, and IL-4 production, except for a tendency to increase the IL-10 levels in the jejunum (Fig. 1C). Moreover, the LAB treatment markedly increased the IL-10 and IL-4 protein levels (Fig. 1D) and had a tendency to increase the IFN- $\gamma$ production in the ileum (Fig. 1D), but the secretion of IL-2 and TNF- $\alpha$ was unaffected in the jejunum and ileum (Fig. 1D). Collectively, these data suggested that the oral administration of $\mathrm{LAB}$ enhances the intestinal immunity in suckling piglets.

\subsection{Effect of LAB on the gut microbiota in suckling piglets}

Microbiota play a critical role in the development of the immune system and induction of the appropriate immune response. ${ }^{26,27}$ As newborns are directly accessible to environmental microbiota, the ecosystem of the gut in newborns is easily shaped by various factors. ${ }^{28}$ Therefore, we determined whether the LAB treatment was able to shape the gut microbiota in suckling piglets. We collected colonic contents from the piglets in the CON and LAB groups for 16S rRNA sequencing to analyze the colon microbiota composition. An average of 12265 \pm 1953 raw reads was generated from each sample. After removing the low-quality sequences, 12179 clean tags were clustered into OTUs. A total of 826 OTUs were found in these two groups, but we failed to observe a difference in the number of OTUs between the CON and LAB groups (Fig. S1A $\dagger$ ). The Venn diagram of bacterial communities showed that the core OTUs of these groups were 473 , and the unique OTUs of the CON and LAB group were 171 and 182, respectively (Fig. S1B $\dagger$ ). We then analyzed the alpha-diversity of the microbiota using Shannon, Chao1, and Simpson indexes and found no remarkable differences between the CON and LAB groups (Fig. 2A). Similarly, principal coordinate analysis (PCOA) failed to show distinct clusters in the beta-diversity of the gut microbiota in these two groups (Fig. 2B and Fig. S1C $\dagger$ ). 

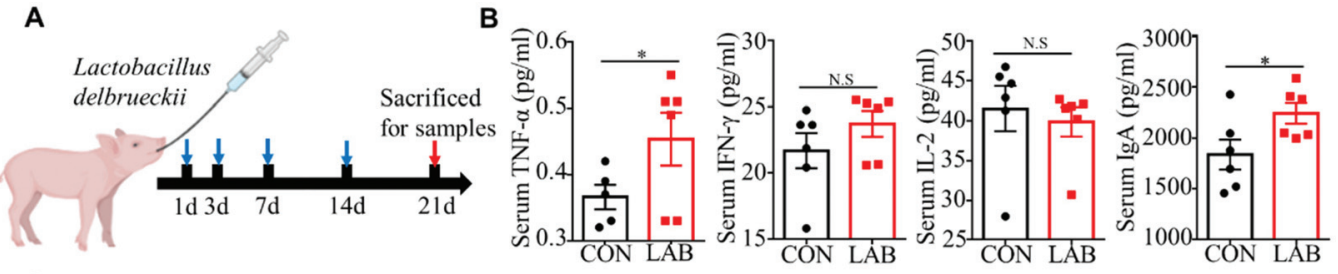

C
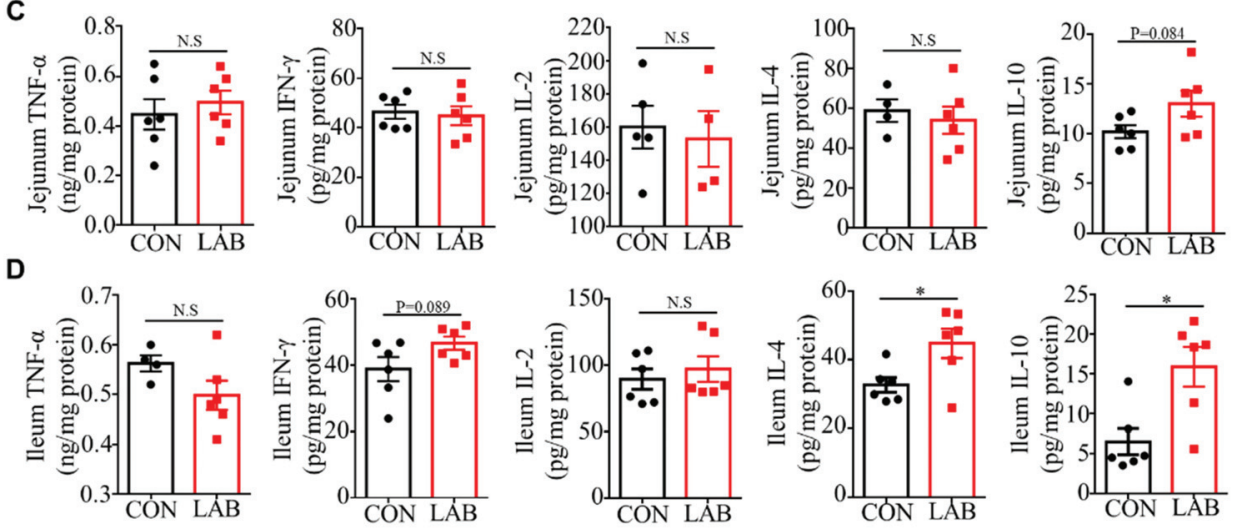

Fig. 1 Oral administration of LAB enhances intestinal immunity in suckling piglets. (A) Schematic diagram of the experimental design. (B) Serum TNF- $\alpha$, IgA, IL-2, and IFN- $\gamma$ levels in piglets from the CON and LAB groups. (C and D) Protein levels of TNF- $\alpha$, IFN- $\gamma$, IL-2, IL-10, and IL-4 in the jejunum (C) and ileum (D) of piglets. Bar graphs show mean \pm SEM. N.S. $P>0.05,{ }^{*} P<0.05, * \star P<0.01$.

The overall microbial composition was further analyzed at the phylum, order, and genus levels. At the phylum level, Firmicutes, Bacteroidetes, and Proteobacteria were the most dominant phyla represented in each group (Fig. 2C), which accounted for more than $96 \%$ of the total bacteria in the colon. However, the $\mathrm{LAB}$ treatment failed to affect the relative abundance of Firmicutes, Bacteroidetes, and Proteobacteria in the colon compared with that in the CON group (Fig. 2D). Similarly, the ratio of Firmicutes to Bacteroidetes was comparable between these two groups (Fig. 2D). At the order level, oral administration of LAB significantly increased the abundance of Lactobacillales, but had no impact on the abundance of other bacterial communities, such as Clostridiales and Bacteroidales (Fig. 2E and F). Although the majority of the genera were comparable between these two groups at the genus level, the LAB treatment potentially increased the relative abundance of Lactobacillus in the colon compared with that in the CON group (Fig. 2G and H). In contrast, oral adminstration of LAB had a tendency to decrease the abundance of Bacteroides compared with that in the CON group (Fig. 2G and $\mathrm{H}$ ). However, the relative abundance of Prevotella was comparable between the CON and LAB groups (Fig. 2G and $\mathrm{H}$ ).

We further performed PICRUSt analysis to evaluate the predicted functions of the microbiota mainly including cellular processes, environmental information processing, genetic information processing, human diseases, metabolism, and organismal systems (Fig. S1D†). KEGG pathways showed that the microbiota-mediated genes were mainly enriched in translation, replication and repair, nucleotide metabolism, amino acid metabolism, energy metabolism, membrane transport, metabolism of cofactors and vitamins, and carbohydrate metabolism. However, oral administration of LAB did not influence these functional processes (Fig. S1D†).

\subsection{Oral administration of LAB induces DC maturation and activation}

Intestinal DCs, functioning as professional antigen presenting cells (APCs), constantly sample the luminal content to monitor for pathogens and commensal bacteria. After sampling antigens, the DCs become mature characterized by higher costimulatory molecule expression, and further mediate the innate and adaptive immune response. ${ }^{29,30}$ Previous data suggested that oral administration of LAB improves intestinal immunity in suckling piglets. ${ }^{16}$ Thus, we hypothesized that LAB may regulate intestinal immunity through inducing DC activation. We first detected the expression of DC maturation markers including CD80, CD86, and MHC-II, and found that oral administration of $\mathrm{LAB}$ dramatically upregulated the CD80, CD86, and $M H C-I I$ expressions in the jejunum and ileum (Fig. 3A and Fig. S2A $\dagger$ ). We also analyzed the expression of chemokine $\mathrm{Ccl} 20$ and its receptor Ccr6 that are critical to DC recruitment. $^{31}$ We observed notably higher Ccl2O and Ccr6 expressions in the jejunum and ileum in the LAB group (Fig. 3B and Fig. S2B $\dagger$ ). Consistently, the LAB treatment tended to increase the serum CCL20 protein levels, but not the CX3CL1 protein levels (Fig. 3C). These in vivo data implied that the LAB treatment may induce DC maturation and activation to regulate intestinal immunity. In support of this, we observed that both heat-killed or live forms of LAB treatment induced higher expressions of CD86, CD80, and MHC-II in DC2.4 cells (Fig. 3D and Fig. S2C†). Interestingly, this effect was more prominent in the live LAB treatment compared to 

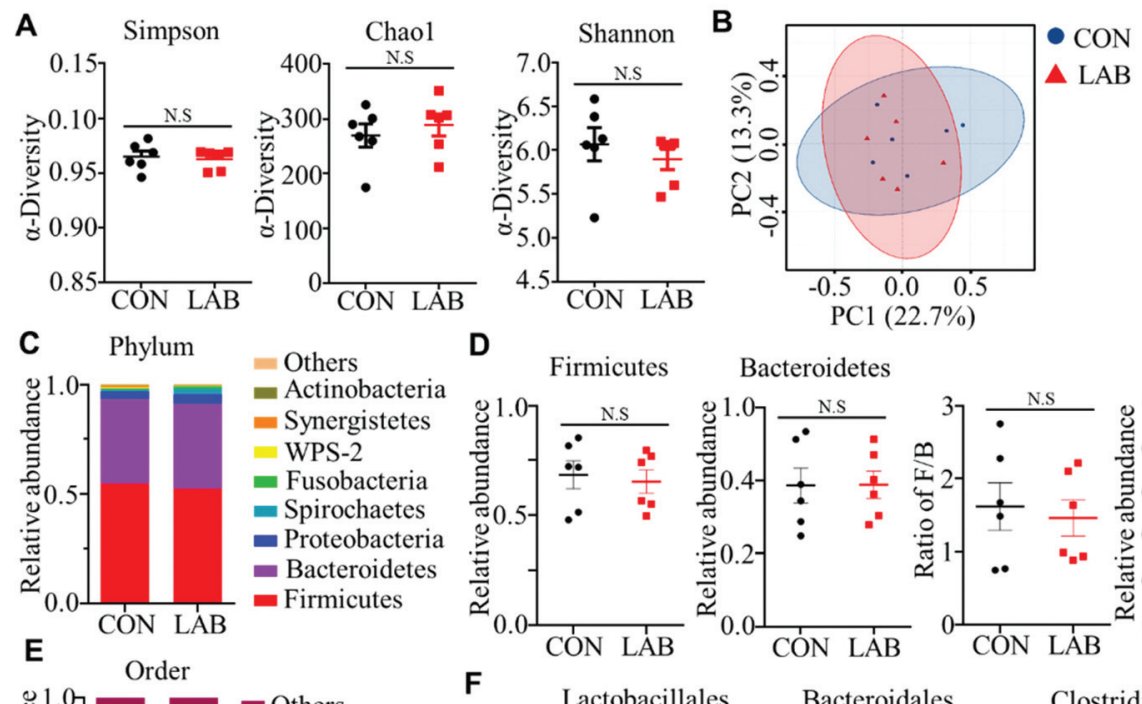

D Firmicutes
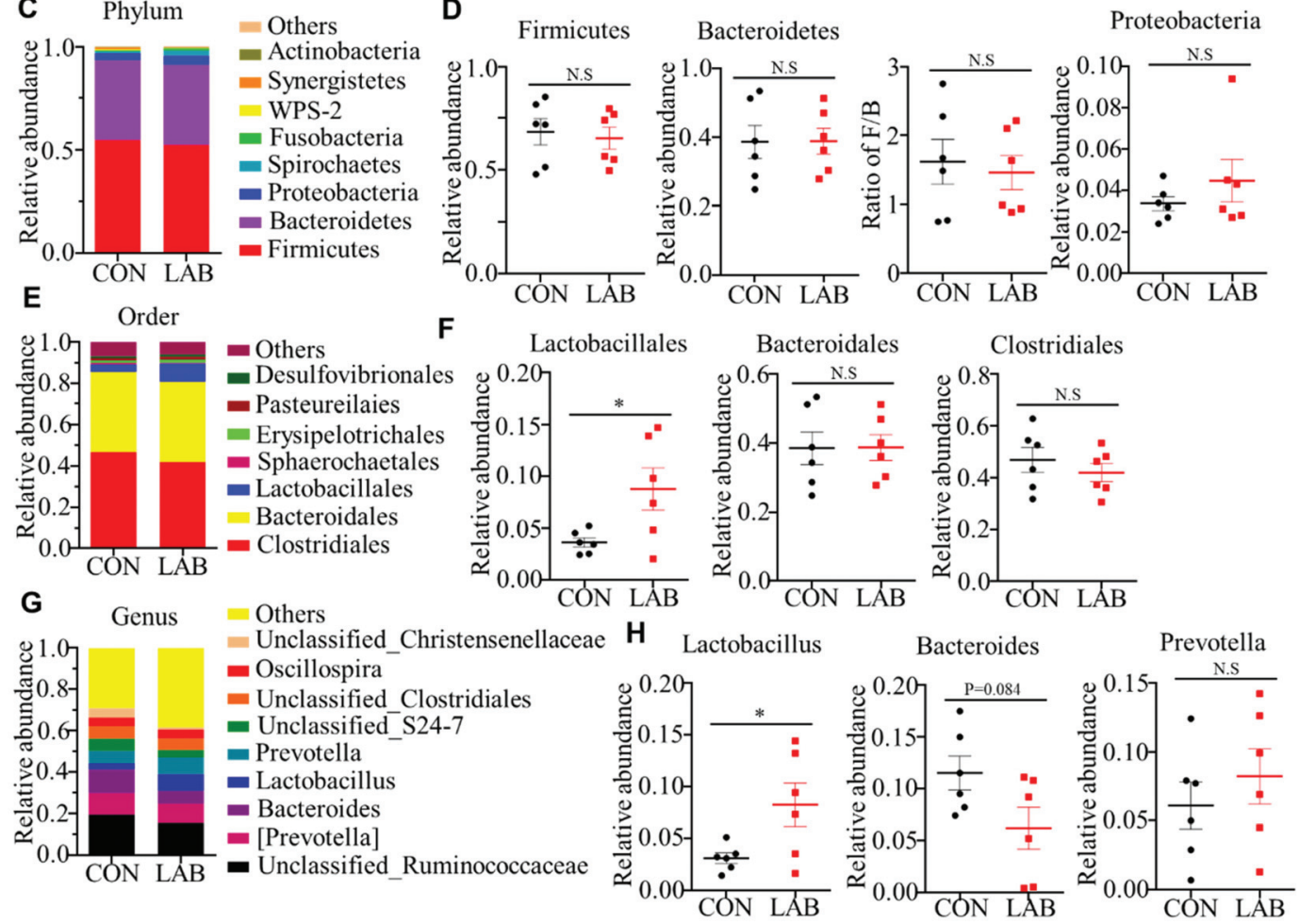

Fig. 2 Effect of LAB on gut microbiota in suckling piglets. (A) Shannon, Chao1 and Simpson index in the $\alpha$-diversity analysis of microbiota in the colon of piglets from the CON and LAB groups. (B) PCoA plot analysis from each sample in the colon of piglets. (C-H) Microbiota compositions at the phylum level $(C$ and $D)$, order level $(E$ and $F)$, and genus level $(G$ and $H)$ in the colon of piglets. Bar graphs show mean $\pm S E M$. N.S. $P>0.05$, ${ }^{*} P<$ $0.05, * \star P<0.01$.

the heat-killed LAB treatment (Fig. 3D). Furthermore, LPS stimulation notably increased IL-12, IL-2, TNF- $\alpha$, and CCL20 production in DC2.4 cells compared with the control group (Fig. 3E). Meanwhile, the treatment with live, but not heatkilled LAB induced IL-12, IL-2, TNF- $\alpha$, and CCL20 production in DC2.4 cells (Fig. 3E). Collectively, these data suggested that LAB may regulate intestinal immunity by inducing DC maturation and activation.

\subsection{LAB induces MAPK and NF-KB activation in DC}

Many studies have shown that TLR2 and TLR4 could sense the presence of microorganisms, which activate MAPK and NF-kB signaling to induce pro-inflammatory cytokine and chemokine production in DCs. ${ }^{29,30}$ Thus, we analyzed the expression of TLR2, TLR4, and MyD88 in the jejunum and ileum of piglets from the CON and LAB groups. We observed that the LAB treatment enhances the expression of TLR2, TLR4, and MyD88 at the mRNA and protein levels in jejunum and ileum (Fig. 4A, B, and Fig. S3A, B广). In line with these in vivo data from jejunum and ileum (Fig. 4A, B, and Fig. S3A, B $\dagger$ ), we confirmed the higher expressions of TLR2, TLR4, and MyD88 at the protein levels in LAB-treated DC2.4 cells (Fig. 4C). To further explore the molecular mechanisms of LAB-mediated DC activation, we determined the expression of Rela, AP1, and Irf3, which are core transcript factors in NF-KB, MAPK, and IRF3 signaling pathways. ${ }^{32}$ We found increased expression of Rela, AP1, Irf3, and Trif in jejunum and ileum in the LAB group (Fig. 4D and Fig. $\mathrm{S}_{\mathrm{C}} \dagger$ ). These in vivo data indicated that LAB might induce NF-KB, MAPK, and IRF3 signaling activation to regulate intestinal immunity of piglets. In support of this, the LAB treatment, with either heat-killed or live form, significantly increased the expressions of p-ERK, p-JNK, p-p38, p-IкB $\alpha$, and p-p65, but not p-IRF3 in DC2.4 cells (Fig. 4E and F). Collectively, these data suggested that LAB might induce DC activation through NF-KB and MAPK signaling to modulate intestinal immunity in suckling piglets. 
A

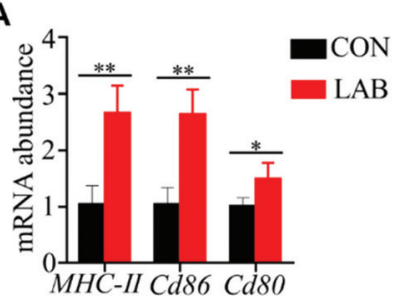

B

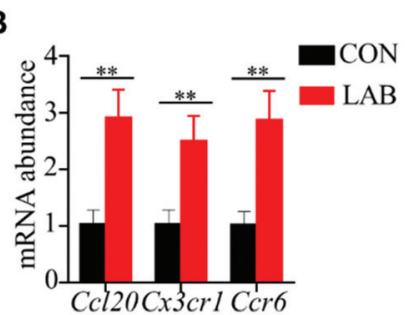

C

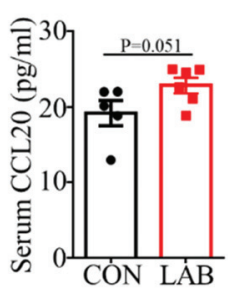

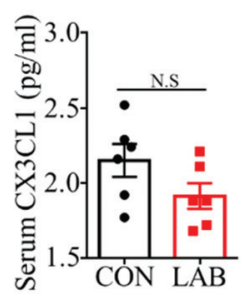

D
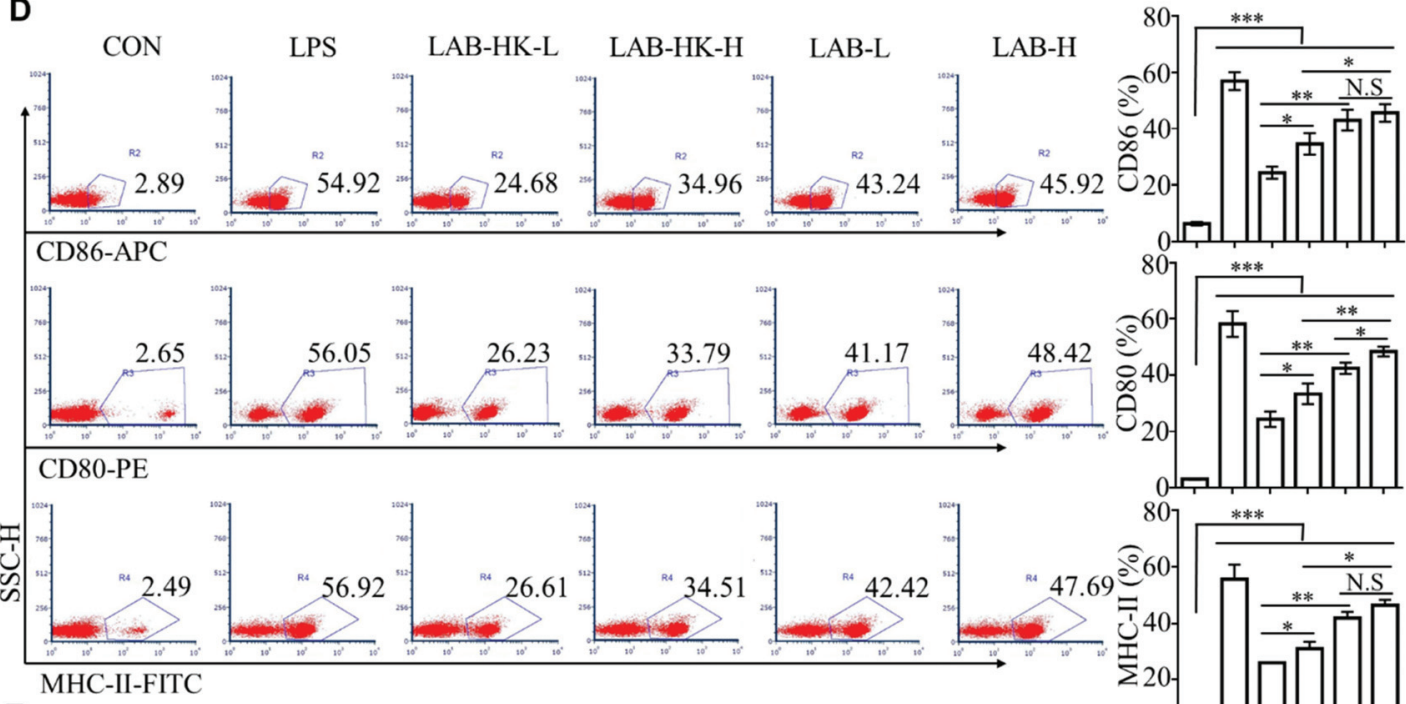

E
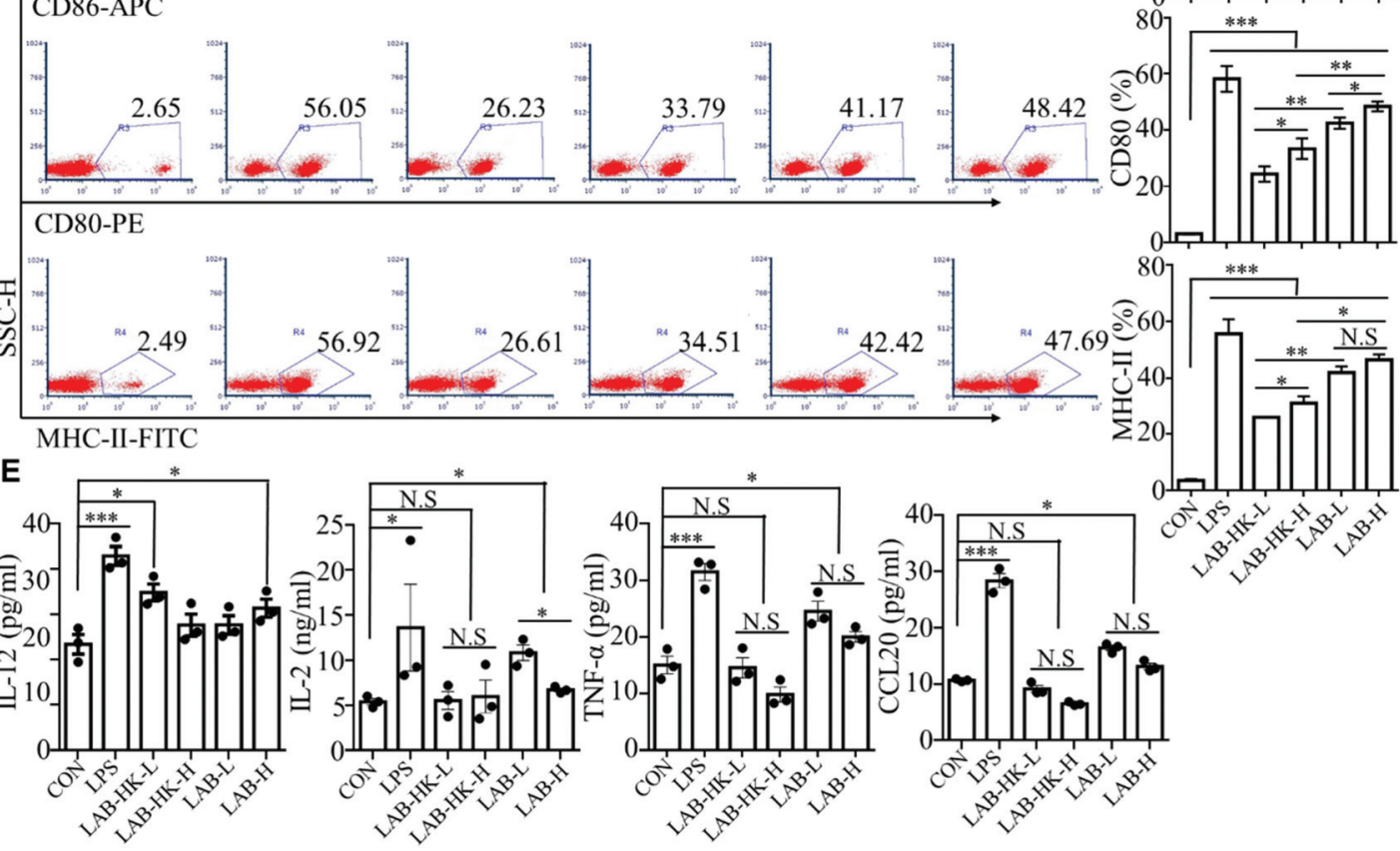

Fig. 3 Oral administration of LAB induces DC maturation and activation. (A and B) The mRNA abundance of CD80, CD86, and MHC-II (A) and Ccl20, Ccr6, and CX3cr1 (B) in the ileum of piglets from the CON and LAB groups. (C) Protein levels of CCL20 and CX3CL1 in the serum of piglets. (D) Flow cytometry analysis of CD80, CD86, and MHC-II in DC2.4 cells stimulated with LPS, low dose of heat-killed (LAB-HK-L) and live LAB (LAB-L), and high dose of heat-killed (LAB-HK-H) and live LAB (LAB-H). (E) ELISA assay for IL-12, IL-2, TNF- $\alpha$, and CCL20 in DC2.4 cells stimulated as in (D). Bar graphs show mean \pm SEM. N.S. $P>0.05,{ }^{\star} P<0.05,{ }^{*} P<0.01$.

3.5 LAB colonization in the suckling period exhibits longlasting positive effects on intestinal immunity of weaned piglets

Early-life microbial colonization is the most important time for shaping intestinal and immune system development, which would exert long-lasting positive effects for enhancing the immune system and preventing the pathogenesis of several diseases. ${ }^{33,34}$ Here, we further tested whether oral administration of $\mathrm{LAB}$ in suckling piglets could exert longlasting effects on intestinal immunity after weaning (Fig. 5A). We observed that the LAB treatment in the suckling period enhanced the serum IL-12 levels on day 28, and had a tendency to increase the serum IgA levels on day 49 (Fig. 5B).
However, the LAB treatment failed to affect the serum IFN- $\gamma$, IL-2, TNF- $\alpha$, and CX3CL1 levels (Fig. S4 $\dagger$ ). Meanwhile, oral administration of LAB in the suckling period dramatically increased the protein levels of TNF- $\alpha$ in jejunum at day 49 and in ileum at day 28 (Fig. 5C and D). The protein levels of IL-4 in the ileum from the LAB group increased at day 28 (Fig. 5D). In contrast, the LAB treatment in the suckling period reduced the IFN- $\gamma$ protein levels in the jejunum (Fig. 5C). However, the protein levels of IL-10 and IL-2 in the jejunum and ileum at days 28 and 49 were comparable between these two groups (Fig. 5C and D). These data suggested that the oral gavage of $\mathrm{LAB}$ in suckling piglets could exert long-lasting effects on intestinal immunity after weaning. 
A

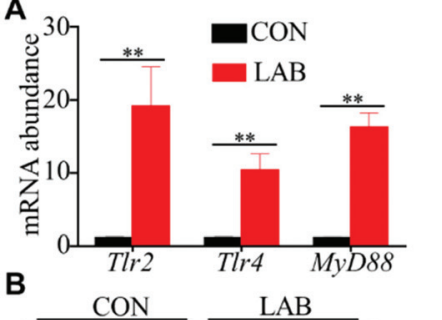

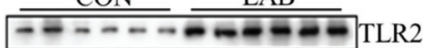

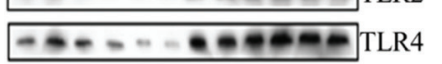

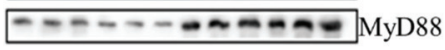

00000000000 $\beta$-actin

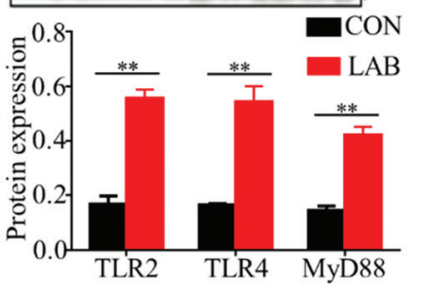

C

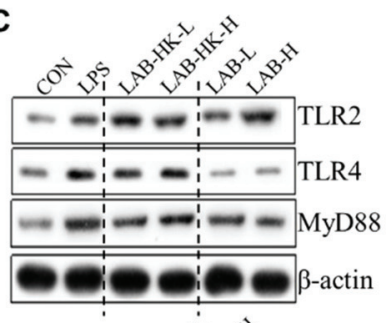

E

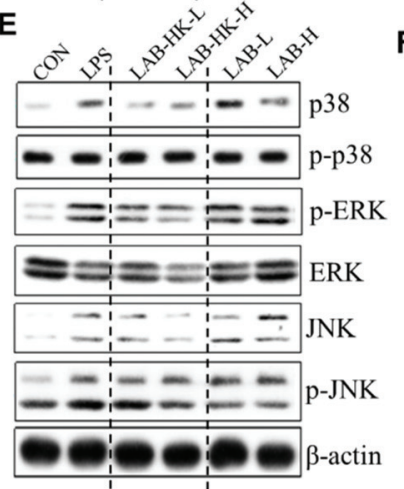

D
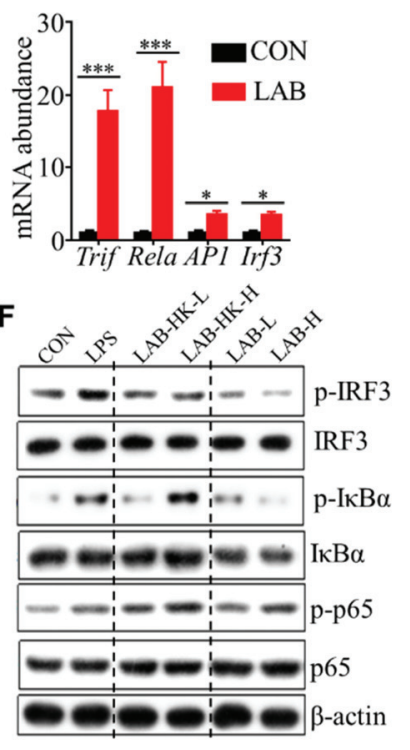

Fig. 4 LAB induces MAPK and NF- $\kappa B$ activation in DC. (A and B) The expression of TLR2, TLR4, and MyD88 at the mRNA (A) and protein (B) levels in the ileum of piglets from the CON and LAB groups. (C) Protein levels of TLR2, TLR4, and MyD88 in DC2.4 cells stimulated with LPS, low dose of heat-killed (LAB-HK-L) and live LAB (LAB-L), and high dose of heat-killed (LAB-HK-H) and live LAB (LAB-H). (D) The mRNA abundance of Trif, Rela, $A P 1$, and Irf3 in the ileum of piglets. (E and F) Immunoblot analysis of $p$-ERK, p-JNK, p-p38, p-IKB $\alpha, p-p 65$, and p-IRF3 in DC2.4 cells stimulated as in (C). Bar graphs show mean \pm SEM. N.S. $P>0.05, * P<0.05, * * P<0.01$.
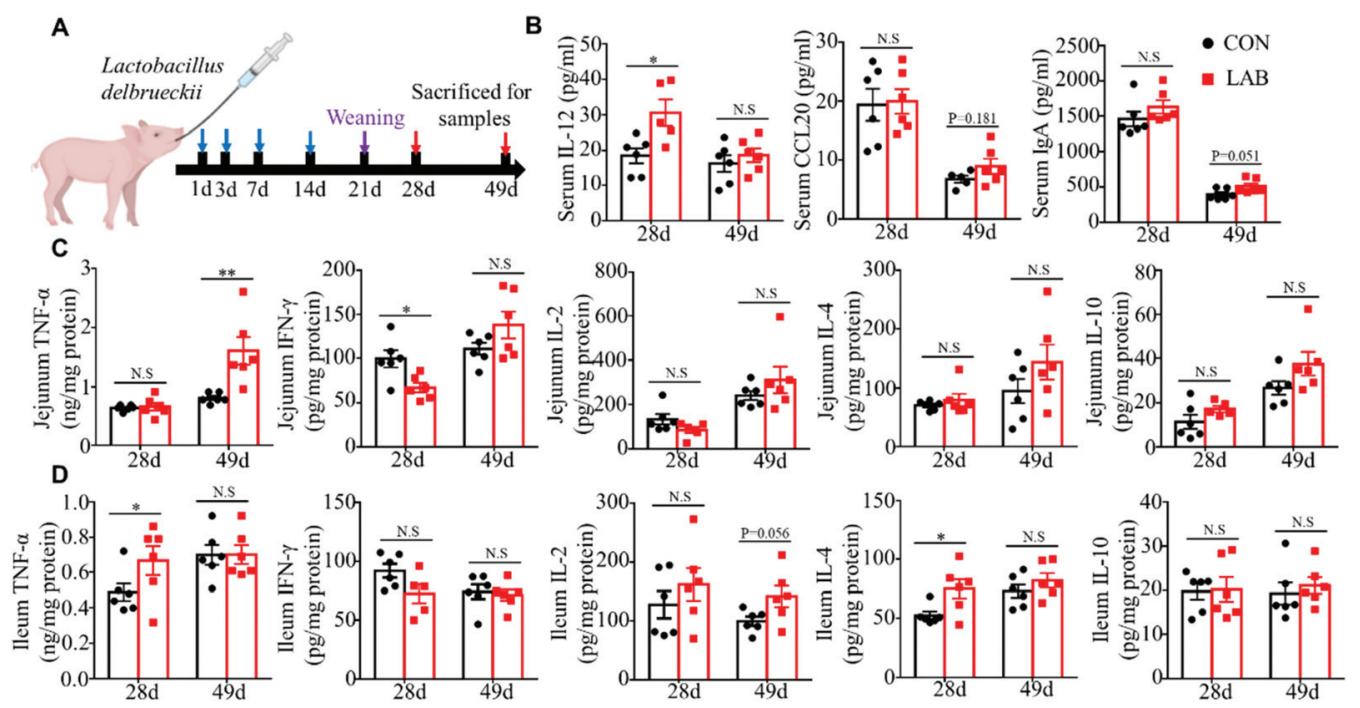

Fig. 5 LAB colonization in the suckling period exhibited long-lasting positive effects on intestinal immunity of the weaned piglet. (A) Schematic diagram of the experimental design. (B) Serum CCL20, IgA, and IL-12 levels in piglets from the CON and LAB groups. (C and D) Protein levels of TNF$\alpha$, IFN- $\gamma$, IL-2, IL-10, and IL-4 in the jejunum (C) and ileum (D) of piglets. Bar graphs show mean \pm SEM. N.S. $P>0.05, * P<0.05, * \star P<0.01$.

\section{Discussion}

Maintenance of gut homeostasis is a guarantee for achieving good performance of humans and animals. The immature development of the gut immune system during early life renders them more susceptible to food and microbial antigens. ${ }^{2,35}$ Accumulating evidence has shown that early-life microbial colonization is essential for gut homeostasis. ${ }^{6,26}$ Myriads of probiotics including Lactobacillus spp., Bifidobacterium spp., and Enterococus spp. contribute to gut immune system development under healthy or pathogenic conditions, which promote the growth performance of animals. ${ }^{5,9,12,36}$ In line with previous studies, we found that oral administration of LAB increases the abundance of the 
Lactobacillus genus and enhances intestinal immune responses in suckling piglets, which provide some evidence to support our previous observation that the LAB treatment improves the growth performance of piglets during the suckling and weaning period. ${ }^{19}$ Of note, we observed that the LAB treatment increases IL-4 and IL-10 production in jejunum and ileum in suckling piglets. IL-10 and IL-4 are pivotal for the proliferation of $\mathrm{B}$ cells and differentiation of these cells into plasma cells, and are key factors that drive the secretion of IgA in the mucosal site. ${ }^{37-39}$ In our previous studies, we found that the LAB treatment increases mucosal sIgA production in the jejunum and ileum from the suckling and weaned piglets. ${ }^{16,18}$ Thus, we speculated that LAB-mediated mucosal sIgA secretion in the intestine is associated with its immunomodulation on IL-4 and IL-10 production, which need to be further investigated.

Intestinal DCs mediate tolerance to food antigens and gut commensal microbiota. ${ }^{30}$ Maturation and differentiation of intestinal DCs can be regulated by many factors including colonization with Lactobacillus spp. or some other probiotic strains. $^{40,41}$ In this study, we found that LAB promotes DC maturation and activation, suggesting a possible link between $\mathrm{LAB}$ administration and improved intestinal immunity. We further found that LAB-induced DC maturation and activation are associated with TLR2/4-mediated NF- $\mathrm{BB}$ and MAPK signaling activation. It is well known that TLR-mediated NF- $\mathrm{B}$ and MAPK signaling is critical to DC maturation and activation, and also crucial to cytokine production and co-stimulatory molecule expression. ${ }^{42-44}$ Indeed, we observed that the LAB treatment increases the co-stimulatory molecules' (CD80 and CD86) expression and cytokines' (IL-12, TNF- $\alpha$, and CCL20) production. In support of our observation, Elawadli et al. also found that the Lactobacillus treatment induces DC maturation and activation. ${ }^{45}$ However, this is in disagreement with some other studies showing that probiotic treatment decreases maturation and inflammatory cytokine production of DCs after LPS or pathogen challenge. ${ }^{46,47}$ Discrepancies of these results are possibly attributed to the different models used in our study because we evaluated the modulatory effects of LAB on DCs in a steady-state. Moreover, some investigations show that the probiotic treatment induces DCs to differentiate into different functional DC subsets, which provide signals to tune the innate and adaptive immune response. ${ }^{48,49}$ However, whether oral administration of LAB could impact intestinal DC differentiation in suckling piglets remains to be studied.

Intriguingly, we observed that the LAB treatment increases the serum IL-12, jejunal and ileal TNF- $\alpha$ levels at days 28 and 49 in the weaned piglets. IL-12 and TNF- $\alpha$ are critical mediators for initiating the innate and adaptive immune response. Many studies have shown that TNF- $\alpha$ and IL-12 play important roles in enhancing the phagocytic and bactericidal effects of macrophages and neutrophils, promoting macrophage and DC activation, and inducing Th1 differentiation and cytotoxic $\mathrm{T}$ lymphocyte activation. ${ }^{50-53}$ Thus, the increased IL-12 and TNF- $\alpha$ levels after weaning in the LAB-treated piglets imply that LAB exhibits long-term immunomodulatory effects on intestinal immunity. Moreover, the administration of probiotics during the early life of humans or mice can prevent or treat diseases such as IBD and allergic diseases. ${ }^{54,55}$ Some researchers ascribe the long-term implications to the effects of probiotics on increasing intestinal microbial diversity during early life. ${ }^{55,56}$ In our study, we found that oral administration of LAB during the suckling period increases the abundance of Lactobacillus species, which would provide rational evidence for the enhanced intestinal immune response and growth performance in the weaning period. ${ }^{19}$

\section{Conclusions}

In summary, we found that oral administration of LAB in suckling piglets enhances intestinal immunity and induces DC maturation and activation. Notably, we found that the immunomodulatory properties of LAB during the suckling period exhibit long-term implications on intestinal immunity during the weaning period. Our data suggest that LAB can be developed as an immunoenhancing biological feed additive.

\section{Ethical statement}

All animal procedures were performed in accordance with the Guidelines for Care and Use of Laboratory Animals of Hunan Agricultural University and approved by the Animal Ethics Committee of Hunan Agricultural University (approval number: 20180722).

\section{Author contributions}

Wei Peng and Xing-Guo Huang designed the experiment. Wei Peng, Ying-Hui Li, Shu-Ling Hou, and Ling-Yuan Yang conducted the experiments. Wei Peng, Li-Xiang Chen, and Jie-Lin Duan performed data analysis. Wei Peng, Guan Yang, Jie-Lin Duan, and Xing-Guo Huang wrote the manuscript.

\section{Conflicts of interest}

The authors declared that there is no conflict of interest.

\section{Acknowledgements}

This study was supported by the National Natural Science Foundation of China (31372322, 31772617, and 31802074), the Nature Science Foundation of Hunan Province (2019JJ50264), the Youth Science Fund Project of Hunan Agricultural University (17QN04), and the Startup Scientific Research Project of Introducing Personnel in Hunan Agricultural University (540490300011). 


\section{References}

1 J. T. Gebhardt, M. D. Tokach, S. S. Dritz, J. M. DeRouchey, J. C. Woodworth, R. D. Goodband and S. C. Henry, Postweaning mortality in commercial swine production II: review of infectious contributing factors, Transl. Anim. Sci., 2020, 4, txaa052.

2 C. R. Stokes, The development and role of microbial-host interactions in gut mucosal immune development, J. Anim. Sci. Biotechnol., 2017, 8, 12.

3 I. Declerck, J. Dewulf, S. Sarrazin and D. Maes, Long-term effects of colostrum intake in piglet mortality and performance, J. Anim. Sci., 2016, 94, 1633-1643.

4 M. E. Sanders, F. Guarner, R. Guerrant, P. R. Holt, E. M. M. Quigley, R. B. Sartor, P. M. Sherman and E. A. Mayer, An update on the use and investigation of probiotics in health and disease, Gut, 2013, 62, 787-796.

5 M. Deng, X. Wu, X. Y. Duan, J. Z. Xu, X. Yang, X. L. Sheng, P. B. Lou, C. L. Shao, C. Lv and Z. Q. Yu, Lactobacillus paracasei L9 improves colitis by expanding butyrate-producing bacteria that inhibit the IL-6/STAT3 signaling pathway, Food Funct., 2021, 12, 10700-10713.

6 Q. Mu, V. J. Tavella and X. M. Luo, Role of Lactobacillus reuteri in Human Health and Diseases, Front. Microbiol., 2018, 9, 757.

7 L. O'Mahony, J. McCarthy, P. Kelly, G. Hurley, F. Luo, K. Chen, G. C. O'Sullivan, B. Kiely, J. K. Collins, F. Shanahan and E. M. Quigley, Lactobacillus and bifidobacterium in irritable bowel syndrome: symptom responses and relationship to cytokine profiles, Gastroenterology, 2005, 128, 541-551.

8 E. Furrie, Is Bifidobacterium a more effective probiotic therapy than Lactobacillus for patients with irritable bowel syndrome?, Nat. Rev. Gastroenterol. Hepatol., 2005, 2, 304305.

9 L. Cervantes-Barragan, J. N. Chai, M. D. Tianero, B. Di Luccia, P. P. Ahern, J. Merriman, V. S. Cortez, M. G. Caparon, M. S. Donia, S. Gilfillan, M. Cella, J. I. Gordon, C. S. Hsieh and M. Colonna, Lactobacillus reuteri induces gut intraepithelial CD4(+)CD8alphaalpha $(+)$ T cells, Science, 2017, 357, 806-810.

10 M. Mohamadzadeh, E. A. Pfeiler, J. B. Brown, M. Zadeh, M. Gramarossa, E. Managlia, P. Bere, B. Sarraj, M. W. Khan, K. C. Pakanati, M. J. Ansari, S. O'Flaherty, T. Barrett and T. R. Klaenhammer, Regulation of induced colonic inflammation by Lactobacillus acidophilus deficient in lipoteichoic acid, Proc. Natl. Acad. Sci. U. S. A., 2011, 108(Suppl. 1), 4623-4630.

11 H. Y. Liu, A. Giraud, C. Seignez, D. Ahl, F. Guo, J. Sedin, T. Walden, J. H. Oh, J. P. van Pijkeren, L. Holm, S. Roos, S. Bertilsson and M. Phillipson, Distinct B cell subsets in Peyer's patches convey probiotic effects by Limosilactobacillus reuteri, Microbiome, 2021, 9, 198.

12 A. Pellon, D. Barriales, A. Pena-Cearra, J. Castelo-Careaga, A. Palacios, N. Lopez, E. Atondo, M. A. Pascual-Itoiz, I. Martin-Ruiz, L. Sampedro, M. Gonzalez-Lopez,
L. Barcena, T. Martin-Mateos, J. M. Landete, R. PradosRosales, L. Plaza-Vinuesa, R. Munoz, B. de Las Rivas, J. M. Rodriguez, E. Berra, A. M. Aransay, L. Abecia, J. L. Lavin, H. Rodriguez and J. Anguita, The commensal bacterium Lactiplantibacillus plantarum imprints innate memory-like responses in mononuclear phagocytes, Gut Microbes, 2021, 13, 1939598.

13 Y. Liu, N. Y. Fatheree, N. Mangalat and J. M. Rhoads, Lactobacillus reuteri strains reduce incidence and severity of experimental necrotizing enterocolitis via modulation of TLR4 and NF-kappaB signaling in the intestine, Am. J. Physiol.: Gastrointest. Liver Physiol., 2012, 302, G608G617.

14 J. Wang, W. Zhang, S. X. Wang, H. Liu, D. Y. Zhang, Y. M. Wang and H. F. Ji, Swine-Derived Probiotic Lactobacillus plantarum Modulates Porcine Intestinal Endogenous Host Defense Peptide Synthesis Through TLR2/MAPK/AP-1 Signaling Pathway, Front. Immunol., 2019, 10, 2691.

15 P. Kanmani and H. Kim, Protective effects of lactic acid Bacteria against TLR4 induced inflammatory response in hepatoma HepG2 cells Through Modulation of Toll-like receptor negative regulators of Mitogen-activated Protein Kinase and NF-kappa B signaling, Front. Immunol., 2018, 9, 1537.

16 Y. Li, S. Hou, W. Peng, Q. Lin, F. Chen, L. Yang, F. Li and X. Huang, Oral Administration of Lactobacillus delbrueckii during the Suckling Phase Improves Antioxidant Activities and Immune Responses after the Weaning Event in a Piglet Model, Oxid. Med. Cell. Longevity, 2019, 2019, 6919803.

17 F. Chen, H. Wang, J. Chen, Y. Liu, W. Wen, Y. Li and X. Huang, Lactobacillus delbrueckii Ameliorates Intestinal Integrity and Antioxidant Ability in Weaned Piglets after a Lipopolysaccharide Challenge, Oxid. Med. Cell. Longevity, 2020, 2020, 6028606.

18 X. L. Wang, Z. Y. Liu, Y. H. Li, L. Y. Yang, J. Yin, J. H. He, D. X. Hou, Y. L. Liu and X. G. Huang, Effects of Dietary Supplementation of Lactobacillus delbrueckii on Gut Microbiome and Intestinal Morphology in Weaned Piglets, Front. Vet. Sci., 2021, 8, 692389.

19 Y. H. Li, S. L. Hou, J. S. Chen, W. Peng, W. Wen, F. M. Chen and X. G. Huang, Oral administration of Lactobacillus delbrueckii during the suckling period improves intestinal integrity after weaning in piglets, J. Funct. Foods, 2019, 63, 103591.

20 F. Chen, J. Chen, Q. Chen, L. Yang, J. Yin, Y. Li and X. Huang, Lactobacillus delbrueckii Protected Intestinal Integrity, Alleviated Intestinal Oxidative Damage, and Activated Toll-Like Receptor-Bruton's Tyrosine KinaseNuclear Factor Erythroid 2-Related Factor 2 Pathway in Weaned Piglets Challenged with Lipopolysaccharide, Antioxidants, 2021, 10, 468.

21 J. L. Duan, J. Yin, W. K. Ren, M. M. Wu, S. Chen, Z. J. Cui, X. Wu, R. L. Huang, T. J. Li and Y. L. Yin, Pyrrolidine dithiocarbamate restores gastric damages and suppressive 
autophagy induced by hydrogen peroxide, Free Radical Res., 2015, 49, 210-218.

22 J. L. Duan, H. Q. He, Y. Yu, T. Liu, S. J. Ma, F. Li, Y. S. Jiang, X. Lin, D. D. Li, Q. Z. Lv, H. H. Ma and X. M. Jia, E3 ligase c-Cbl regulates intestinal inflammation through suppressing fungi-induced noncanonical NF-kappaB activation, Sci. Adv., 2021, 7, eabe5171.

23 J. Yin, Y. Li, H. Han, S. Chen, J. Gao, G. Liu, X. Wu, J. Deng, Q. Yu, X. Huang, R. Fang, T. Li, R. J. Reiter, D. Zhang, C. Zhu, G. Zhu, W. Ren and Y. Yin, Melatonin reprogramming of gut microbiota improves lipid dysmetabolism in high-fat diet-fed mice, J. Pineal Res., 2018, 65, e12524.

24 J. Yin, Y. Li, H. Han, J. Ma, G. Liu, X. Wu, X. Huang, R. Fang, K. Baba, P. Bin, G. Zhu, W. Ren, B. Tan, G. Tosini, X. He, T. Li and Y. Yin, Administration of Exogenous Melatonin Improves the Diurnal Rhythms of the Gut Microbiota in Mice Fed a High-Fat Diet, mSystems, 2020, 5, e00002-e00020.

25 Y. Liu, Q. Lin, X. Huang, G. Jiang, C. Li, X. Zhang, S. Liu, L. He, Y. Liu, Q. Dai and X. Huang, Effects of Dietary Ferulic Acid on the Intestinal Microbiota and the Associated Changes on the Growth Performance, Serum Cytokine Profile, and Intestinal Morphology in Ducks, Front. Microbiol., 2021, 12, 698213.

26 H. J. Flint, K. P. Scott, P. Louis and S. H. Duncan, The role of the gut microbiota in nutrition and health, Nat. Rev. Gastroenterol. Hepatol., 2012, 9, 577-589.

27 A. M. Valdes, J. Walter, E. Segal and T. D. Spector, Role of the gut microbiota in nutrition and health, Br. Med. J., 2018, 361, k2179.

28 C. Y. L. Chong, F. H. Bloomfield and J. M. O'Sullivan, Factors Affecting Gastrointestinal Microbiome Development in Neonates, Nutrients, 2018, 10, 274.

29 M. Rescigno and A. Di Sabatino, Dendritic cells in intestinal homeostasis and disease, J. Clin. Invest., 2009, 119, 2441-2450.

30 A. J. Stagg, Intestinal Dendritic Cells in Health and Gut Inflammation, Front. Immunol., 2018, 9, 2883.

31 E. Schutyser, S. Struyf and J. Van Damme, The CC chemokine CCL20 and its receptor CCR6, Cytokine Growth Factor Rev., 2003, 14, 409-426.

32 T. H. Mogensen, Pathogen recognition and inflammatory signaling in innate immune defenses, Clin. Microbiol. Rev., 2009, 22, 240-273.

33 I. Laforest-Lapointe and M. C. Arrieta, Patterns of Early-Life Gut Microbial Colonization during Human Immune Development: An Ecological Perspective, Front. Immunol., 2017, 8, 788.

34 P. D. Houghteling and W. A. Walker, Why Is Initial Bacterial Colonization of the Intestine Important to Infants' and Children's Health?, J. Pediatr. Gastroenterol. Nutr., 2015, 60, 294-307.

35 H. J. Rothkotter, Anatomical particularities of the porcine immune system-a physician's view, Dev. Comp. Immunol., 2009, 33, 267-272.
36 J. L. Round, S. M. Lee, J. Li, G. Tran, B. Jabri, T. A. Chatila and S. K. Mazmanian, The Toll-like receptor 2 pathway establishes colonization by a commensal of the human microbiota, Science, 2011, 332, 974-977.

37 T. Defrance, B. Vanbervliet, J. Pene and J. Banchereau, Human recombinant IL-4 induces activated B lymphocytes to produce IgG and IgM, J. Immunol., 1988, 141, 2000-2005.

38 F. Rousset, E. Garcia, T. Defrance, C. Peronne, N. Vezzio, D. H. Hsu, R. Kastelein, K. W. Moore and J. Banchereau, Interleukin 10 is a potent growth and differentiation factor for activated human B lymphocytes, Proc. Natl. Acad. Sci. U. S. A., 1992, 89, 1890-1893.

39 D. A. Lebman and R. L. Coffman, The effects of IL-4 and IL-5 on the IgA response by murine Peyer's patch B cell subpopulations, J. Immunol., 1988, 141, 2050-2056.

40 F. Sakai, T. Hosoya, A. Ono-Ohmachi, K. Ukibe, A. Ogawa, T. Moriya, Y. Kadooka, T. Shiozaki, H. Nakagawa, Y. Nakayama and T. Miyazaki, Lactobacillus gasseri SBT2055 Induces TGF-beta Expression in Dendritic Cells and Activates TLR2 Signal to Produce IgA in the Small Intestine, PLoS One, 2014, 9, e105370.

41 S. B. Ghavami, H. A. Aghdaei, D. Sorrentino, S. Shahrokh, M. Farmani, F. Ashrafian, M. P. Dore, S. K. A. Raftar, S. M. Khoramjoo and M. R. Zali, Probiotic-Induced Tolerogenic Dendritic Cells: A Novel Therapy for Inflammatory Bowel Disease?, Int. J. Mol. Sci., 2021, 22, 8274.

42 T. H. Mogensen, Pathogen Recognition and Inflammatory Signaling in Innate Immune Defenses, Clin. Microbiol. Rev., 2009, 22, 240-273.

43 T. Kawai and S. Akira, Signaling to NF-kappa B by Toll-like receptors, Trends Mol. Med., 2007, 13, 460-469.

44 M. Dalod, R. Chelbi, B. Malissen and T. Lawrence, Dendritic cell maturation: functional specialization through signaling specificity and transcriptional programming, EMBO J., 2014, 33, 1104-1116.

45 I. Elawadli, J. T. Brisbin, B. A. Mallard, M. W. Griffiths, M. Corredig and S. Sharif, Differential effects of lactobacilli on activation and maturation of mouse dendritic cells, Benefic. Microbes, 2014, 5, 323-334.

46 A. L. Hart, K. Lammers, P. Brigidi, B. Vitali, F. Rizzello, P. Gionchetti, M. Campieri, M. A. Kamm, S. C. Knight and A. J. Stagg, Modulation of human dendritic cell phenotype and function by probiotic bacteria, Gut, 2004, 53, 16021609.

47 S. A. Esmaeili, M. Mahmoudi, Z. Rezaieyazdi, M. Sahebari, N. Tabasi, A. Sahebkar and M. Rastin, Generation of tolerogenic dendritic cells using Lactobacillus rhamnosus and Lactobacillus delbrueckii as tolerogenic probiotics, J. Cell. Biochem., 2018, 119, 7865-7872.

48 Z. D. Zhang, J. J. Li, W. C. Zheng, G. Zhao, H. Zhang, X. F. Wang, Y. Q. Guo, C. Qin and Y. Shi, Peripheral Lymphoid Volume Expansion and Maintenance Are Controlled by Gut Microbiota via RALDH(+) Dendritic Cells, Immunity, 2016, 44, 330-342. 
49 P. Ricciardi-Castagnoli, Sensing the gut microbiota, Nat. Immunol., 2020, 21, 704-705.

50 W. W. Lin and M. Karin, A cytokine-mediated link between innate immunity, inflammation, and cancer, J. Clin. Invest., 2007, 117, 1175-1183.

51 J. Šedý, V. Bekiaris and C. F. Ware, Tumor Necrosis Factor Superfamily in Innate Immunity and Inflammation, Cold Spring Harbor Perspect. Biol., 2015, 7, a016279.

52 J. M. Trevejo, M. W. Marino, N. Philpott, R. Josien, E. C. Richards, K. B. Elkon and E. Falck-Pedersen, TNFalpha-dependent maturation of local dendritic cells is critical for activating the adaptive immune response to virus infection, Proc. Natl. Acad. Sci. U. S. A., 2001, 98, 1216212167.
53 G. Trinchieri, Interleukin-12 and the regulation of innate resistance and adaptive immunity, Nat. Rev. Immunol., 2003, 3, 133-146.

54 J. Lee, D. Seto and L. Bielory, Meta-analysis of clinical trials of probiotics for prevention and treatment of pediatric atopic dermatitis, J. Allergy Clin. Immunol., 2008, 121, 116121.e111.

55 H. Szajewska, Short- and long-term effects of probiotics administered early in life, Nestle Nutr. Workshop Ser., Pediatr. Program, 2011, 68, 65-78, discussion 78-82.

56 T. L. Nowland, R. N. Kirkwood and J. R. Pluske, Review: Can early-life establishment of the piglet intestinal microbiota influence production outcomes?, Animal, 2021, 100368, DOI: 10.1016/j.animal.2021.100368. 